\title{
¿Por qué la Fatiga Afecta a los Marineros más que a Otros? Una Voz en el Debate sobre Derechos Humanos ${ }^{1}$
}

\section{Barbara Stępień ${ }^{2}$}

Sumario: Introducción II. Delimitación del problema. III. La fatiga de la gente del mar. IV. Dotación mínima de seguridad. V. Conclusiones

Resumen: En este artículo se sostiene que la profesión de marinero es compleja y peligrosa, debido al alto número de factores que contribuyen a la fatiga (resultado de la forma de vivir y trabajar en el mar). La fatiga también debe considerarse un factor que contribuye sustancialmente al número de accidentes en el mar. Se analizan diversas normas que regulan medidas concernientes a la fatiga en el mar. La implementación de regulaciones internacionales en los sistemas nacionales puede no ser suficiente para resolver muchos de los problemas de los marineros y la industria marítima. A pesar de los estándares internacionales, no se puede apreciar un decremento de la fatiga de los marineros ni tampoco una reducción del número de accidentes. Se concluye que los estándares internacionales y nacionales frecuentemente no se encuentran diseñados para las condiciones de vida y trabajo en el mar y se sostiene que el problema de fatiga a bordo es causado también por un inapropiado manejo de las embarcaciones.

Palabras clave: Derecho marítimo, dotación mínima de seguridad.

Abstract: In this article the author points out that a seafarer's profession is a risky and difficult profession, mainly due to a number of factors (resulting from the way of working and living at sea) contributing to fatigue. Fatigue is also considered to be a substantial contributing factor to the number of accidents at sea. The author analyzes various international regulations concerning fatigue at the sea. The article argues that the implementation of international regulations to the national legal systems might be not sufficient to resolve many problems of mariners and maritime industry. Despite the international standards, a significant decrease of fatigue of seafarers and the number of accidents at sea is not noticeable. It is concluded that international and national standards are often unsuited to the conditions of living and working at sea and that the inappropriate management on ships causes the problem of fatigue on boards also.

Key Words: maritime law, fatigue, safe manning

\footnotetext{
1 Traducción del polaco de Mauro Arturo Rivera León. Este artículo apareció publicado como "Dlaczego zmęczenie dotyka ludzi morza bardziej niż innych? głos w dyskusji o prawach człowieka", Gentes et Nationes, Bielsko-Biała, núm. 8, 2013, págs. 129-136.

2 Capitán de Yate 200GT. Doctora en Derecho con especialidad en derecho internacional público por la Universidad Jaguelónica (Cracovia). Investigadora visitante en el Instituto Max Planck de Derecho Público Comparado y Derecho Internacional (2014). Su más reciente libro es Morska $i$ oceaniczna żegluga jachtowa w świetle prawa międzynarodowego publicznego (Velero marítimo y oceánico a la luz del Derecho Internacional Público), 2013.
} 


\section{Introducción}

Recientemente ha despertado un gran interés científico por temas como los derechos humanos y el terrorismo $\mathrm{o}$ el derecho humanitario. Por consiguiente, en los últimos años, ha habido muchas publicaciones sobre el respeto a los derechos humanos, su violación durante los conflictos armados o la discriminación contra las mujeres. Muchos foros nacionales e internacionales se ocupan de la unión civil entre personas del mismo sexo, la adopción por parejas homosexuales o la ideología de género. Dichas cuestiones son motivo de preocupación para muchas organizaciones gubernamentales y no gubernamentales, medios de comunicación y gran parte de la población.

Sin embargo, en todo momento, el debate sobre los derechos y deberes del "hombre", su seguridad y modo de vida, olvida a un grupo social y profesional numeroso, a saber, los marineros o -en otras palabras- la gente del mar. Por tanto, vale la pena alzar la voz sobre este tema.

\section{Delimitación del problema}

El factor humano en el mar es un tema complejo y multidimensional que afecta tanto la seguridad de las personas (tripulación y pasajeros), la propiedad privada (aproximadamente el $90 \%$ de los bienes se transportan a través del mar), las unidades marítimas (naves) y la protección del medio ambiente marino (más del $71 \%$ de la superficie de la tierra se encuentra ocupada por océanos, el océano mundial afecta a más del $90 \%$ de la biósfera y los cambios en las aguas marinas y su ecosistema tiene efectos nocivos en la tierra ${ }^{3}$ ).

El trabajo de la gente en el mar es pesado y demandante debido a, entre otros factores, el modo (rotativo) de guardias, la necesidad de trabajar durante la noche, las frecuentes condiciones climáticas adversas y la imposibilidad de descansar en casa, teniendo que soportar meses de separación de los seres queridos.

De igual forma, los barcos funcionan por lo regular con el mínimo número de personal necesario y cada empleado tiene más funciones de las que le

3 Łukaszuk, Leonard, "Międzynarodowa ochrona środowiska morskiego - głowne problemy, system regulacji oraz zasady i formy wspołpracy (zarys ogolny)", Studia Ecologiae et Bioethicae, Varsovia, núm. 1, 2003, p. 249. 
corresponderían con una tripulación mayor (y la cantidad de trabajo a realizar no se reduce).

\section{La Fatiga de la Gente del Mar}

La fatiga es uno de los mayores problemas relacionados con la industria de la navegación y el trabajo marítimo. Las condiciones de este tipo de trabajo se consideran extremadamente peligrosas y requieren de una atención y cuidado sostenido ${ }^{4}$. Aunado a ello, el problema de la fatiga se exacerba por el hecho de que afecta a todas las personas que viven y trabajan en el mar, sin importar su función, entrenamiento, habilidades, conocimiento o experticia ${ }^{5}$. Se cree que la fatiga es un factor que influye significativamente en el $11-23 \%$ de los accidentes en el mar, como las colisiones o la navegación en aguas poco profundas $^{6}$.

De acuerdo a la resolución de la Organización Marítima Internacional A.772 (18) sobre los Factores que contribuyen a la Fatiga desde el punto de vista de la dotación mínima y la seguridad (Fatigue Factors in Manning and Safety), no existe una definición universal de fatiga. La fatiga sólo puede ser descrita en términos generales e indicar factores relacionados o categorías. El propósito de dicha resolución es despertar la conciencia en torno a la complejidad del problema de la fatiga, así como incentivar a que ésta sea tomada en cuenta como un factor en las decisiones concernientes a los barcos.

El segundo punto del anexo a la resolución A.772 (18) sostiene que la fatiga conduce a una degradación del desempeño humano, reduciendo los

\footnotetext{
4 Marine Accident Investigation Branch, Annual report, 1999, Southampton, p. 5. Disponible en internet:

http://www.maib.gov.uk/cms_resources.cfm?file=/annual\%20report\%201999.pdf (Última consulta 15.02.2013).

5 IMO MSC/Circ. 1014, 12 de junio de 2001, Guidance on fatigue mitigation and management, Anexo "Guidelines on fatigue, Introduction, Foreword", p. 1.

6 Sin embargo, debe de notarse que estos datos pueden ser subestimaciones debido a la falta de servicios apropiados para reportar todos los accidentes que ocurren. El factor fatiga jugó un papel relevante en el choque del buque petrolero Exxon Valdez que transportaba petróleo crudo desde Valdez, Alaska. El 24 de marzo de 1989, la nave entró en el Estrecho del Príncipe Guillermo produciendo un desastre ambiental debido a la filtración en el mar de un enorme cargamento de petróleo crudo. Como se demostró más adelante por la investigación realizada por la National Transportation Safety Board (NTSB), la fatiga fue considerada la causa del error cometido por el tercer oficial que estaba de guardia y, por tanto, causa directa del accidente (Ross, Jonathan, Human factors for Naval Marine vehicle design and operation, Bodmin, Ashgate, 2009, p. 59).
} 
reflejos físicos y mentales y disminuye la capacidad de hacer juicios 0 valoraciones racionales. La fatiga puede ser causada por factores como prolongados periodos de actividad física y mental, un descanso inadecuado, factores ambientales inadecuados, factores fisiológicos, estrés y otros factores psicológicos.

En el caso de los marineros (de acuerdo al punto 3 del anexo a la resolución) entre las causas más identificadas y documentadas de fatiga se encuentra la escasa calidad del descanso, el exceso de trabajo, el ruido y las relaciones interpersonales. Los factores que conducen a lo anterior son múltiples y variados, debiendo tomarse en cuenta que el aumento de factores específicos variará dependiendo de las condiciones de cada navío en concreto. Por tanto, algunos factores serán más sencillos de controlar y superar que otros.

De acuerdo al punto 4.1 del anexo a la resolución, la prevención de la fatiga en términos de planificación del trabajo, descanso, procedimientos de guardia y delimitación de deberes, puede ser mayormente implementada mediante un correcto manejo desde la costa y apropiadas técnicas de dirección en la nave. Se considera que la administración marítima juega un papel de gran relevancia en la creación de legislación que favorezca la adopción, aplicación y el cumplimiento de los convenios internacionales relativos a las cuestiones anteriores. Durante la creación de nuevas directrices y recomendaciones, debe tenerse en cuenta la relación entre el trabajo y el periodo de descanso, así como los aspectos relacionados a la extensión del viaje, el tiempo de estancia en puerto, tiempo de trabajo individual de los miembros de la tripulación, periodos de responsabilidad o los sistemas de guardia. También es importante que la gestión proporcione un acceso claro y conciso a los criterios sobre el manejo de la nave. Estos requerimientos deben de permitir a la tripulación conocer los procedimientos operativos y familiarizarse con las condiciones prevalecientes en la nave, duración y destino del viaje, características de la carga, así como métodos de comunicación interna y externa. Una administración adecuada debe garantizar que los miembros de la tripulación recién llegados tendrán un descanso adecuado después de su viaje hacia la nave antes de comenzar a desempeñar su trabajo. 
También es importante (de acuerdo al punto 4.2 del anexo a la resolución) durante la modificación o diseño de las naves, el incluir las recomendaciones existentes en estándares y publicaciones sobre factores específicos de la nave (i.e. nivel de automatización, fiabilidad del equipo, movimiento característico, vibración, temperatura, ruido, calidad del medio de trabajo y vida, características y requisitos de la carga estructura de la nave). Adicionalmente, deben implementarse medidas ergonómicas que prevengan aún más el factor fatiga.

Un factor importante (de acuerdo al punto 4.3 del anexo a la resolución) de contramedidas a la fatiga en el mar es un nivel apropiado y meticuloso de entrenamiento, capacidad de los servicios, incluyendo condiciones de salud, así como experiencia adecuada, calidad y cualificaciones de la tripulación.

De acuerdo al punto 4.3.2 del anexo a la resolución, es necesario también estar conscientes de los potenciales problemas que pueden surgir a raíz del empleo en un mismo barco de tripulaciones multinacionales. Esta situación puede dar lugar a formación de barreras idiomáticas y de contexto social, cultural y de credo (religioso) que conlleva al aislamiento de los individuos. Ello, a su vez, puede afectar negativamente la seguridad a bordo. También se debe prestar especial atención a las cuestiones relacionadas con las relaciones interpersonales, la soledad, privación social y responsabilidades crecientes de sobrecarga laboral, que son fenómenos que pueden ocurrir debido al bajo número de tripulantes. Curiosamente, un elemento que también debe ser considerado en el contexto de factores que contribuyen a la fatiga, es el aburrimiento. En consecuencia, es necesario proveer una apropiada estimulación psíquica y física.

Adicionalmente (punto 4.4 de la resolución) se ha indicado factores medioambientales externos como el clima, las condiciones portuarias, condiciones del hielo y densidad del tráfico, como factores que en gran medida contribuyen a la fatiga.

Por ello, es extremadamente importante el cumplimiento del Convenio sobre el Trabajo Marítimo ${ }^{7}$ (2006) -MLC- en lo relativo al máximo de horas laborales y mínimo de horas de descanso. De acuerdo a la norma A 2.3 MLC

7 Convenio sobre el Trabajo Marítimo (MLC), 2006. Ratificado por Polonia (Diario Oficial Dziennik Ustaw Rzeczypospolitej Polskiej-, núm. 222, disposición 1324) y, por tanto, parte integrante del sistema jurídico polaco. 
2006, los estados firmantes deben prever en su legislación interna ${ }^{8}$ un periodo de trabajo en el mar que no exceda las 14 horas en un periodo de 24 horas y 72 horas en un periodo de siete días; o, de igual forma, un periodo mínimo de descanso no menor a 10 horas en un periodo de 24 horas y 77 horas de descanso en un periodo de siete días.

\section{Dotación Mínima de Seguridad}

Uno de los problemas directamente relacionado con la fatiga en el mar es la dotación de unidades respecto a las anteriormente mencionadas condiciones de la tripulación. Paradójicamente, hoy en día, cuando los mares y océanos son navegados en mayor medida y por naves más grandes, los dueños de naves intentan reducir los miembros de la tripulación al mínimo. Como en cualquier otra aventura de negocios, aquí la búsqueda por un mayor beneficio, lleva a reducir costos en el personal.

Debido al avance tecnológico de los buques, una parte sustancial puede ser cubierta con un promedio de 12 a 17 miembros de tripulación, mientras que, por ejemplo, en los años cincuenta del siglo $\mathrm{XX}$ un típico elenco incluía a un promedio de 40 a 50 personas ${ }^{9}$.

Reducir el número de miembros de la tripulación afecta adversamente a la seguridad de las naves al incrementar la fatiga entre los marineros y, por tanto, los riesgos de accidentes. El análisis de los datos sobre accidentes en el mar indica que en veinte años ha habido un sistemáticamente creciente número de accidentes en los cuales la gente del mar ha comprometido su salud e incluso sus vidas. Este tiempo coincide con la también sistemática disminución constante de los miembros de la tripulación a bordo ${ }^{10}$. Sin embargo, debido a las diferentes formas de registro de accidentes en el mar en el mundo, es difícil establecer una relación causal directa entre la tripulación de la cabina y el

8 De acuerdo a los datos disponibles en enero 9 de 2014, los siguientes 56 países son partes del Convenio sobre Trabajo Marítimo (MLC) del 2006.

http://www.ilo.org/dyn/normlex/en/f?p=NORMLEXPUB:11300:0::NO:11300:P11300_ INSTRUMENT_ID: 312331: NO> (ÚLTIMA CONSULTA 01.03.2014).

9 Corbett, Peter, A Modern Plague of Pirates, Reino Unido, Offshore and Marine Publications, 2009, p. 35; Committee on the Effect of Smaller Crews on Maritime Safety, Marine Board, Commission on Engineering and Technical Systems, National Research Council, Crew Size and Maritime Safety, Washington D.C. 1990, p. 1.

10 Committee on the Effect of Smaller Crews on Maritime Safety, Marine Board, Commission on Engineering and Technical Systems, National Research Council, op. cit., p. 85. 
aumento del número de accidentes. De cualquier forma, vale la pena prestar atención a estas tendencias y tratar este fenómeno como un contribuyente a los accidentes en el mar.

La convención básica para la dotación mínima de seguridad es el Convenio Internacional para la Seguridad de la Vida Humana en el Mar (International

Convention for the Safety of Life at Sea - SOLAS), de 1974, en su forma enmendada ${ }^{11}$.

De acuerdo con la regulación 14.1 del Capítulo $\mathrm{V}$ del anexo a la convención SOLAS del 74', los estados firmantes ${ }^{12}$ de la convención deben garantizar reglas respectos a sus flotas que aseguren que cada embarcación se encuentra bajo control de una tripulación competente y numerosa.

Debido a que el apropiado número de miembros de la tripulación es tan importante para la seguridad de la nave, en 1999 la Organización Marítima Internacional adoptó la resolución A.890 (21) Principios relativos a la Dotación Mínima de Seguridad (Principles of Safe Manning ${ }^{13}$ ). Esta resolución muestra que la dotación mínima de seguridad se encuentra en función del número de marineros con experiencia y cualificación necesarios para la seguridad de la nave, tripulación, pasajeros, bienes, propiedad y protección del medio ambiente marítimo y que la capacidad de los marineros de actuar de acuerdo a estos requerimientos también depende de su entrenamiento, trabajo, tiempo de esparcimiento, seguridad, salud, higiene y dieta apropiada.

11 Convenio Internacional para la Seguridad de la Vida Humana en el Mar (SOLAS), 1974. Ratificado por Polonia (Diario Oficial - Dziennik Ustaw Rzeczypospolitej Polskiej-, 1984, núm. 61, disposición 318) y, por tanto, parte integrante del sistema jurídico polaco.

12 De acuerdo con los datos disponibles a 9 de enero de 2014, 162 países son partes en el Convenio Internacional para la Seguridad de la Vida Humana en el Mar (SOLAS). El tonelaje bruto de la flota mercando de estos países representa en su conjunto aproximadamente el $99.20 \%$ de la flota mercante mundial. International Maritime Organization, Status of multilateral Conventions and instruments in respect of which the International Maritime Organization or its Secretary-General performs depositary or other functions, 9 de enero de 2014, p. 18. Disponible en internet:

<http://www.imo.org/About/Conventions/StatusOfConventions/Documents/Status\%20-

$\% 202014 \% 20 N e w \% 20$ Version.pdf> (última consulta 1.03.2014).

13 De acuerdo con la regulación 14.2 del capítulo V del anexo a la Convención SOLAS'74, cada nave, conforme a la relación contenida en el capítulo I, deberá tener un certificado de dotación mínima de seguridad de la nave o su equivalente. Esto es, información acerca del número de tripulantes que se reputan necesarios para garantizar la seguridad de acuerdo a las provisiones del párrafo 1. 
De acuerdo con el párrafo primero del anexo I a la resolución A.890 (21), enmendada ésta por la resolución A.955 (23), la dotación mínima de seguridad debe hacerse de acuerdo a las siguientes reglas ${ }^{14}$.

- Mantener segura la navegación, ingeniería y radio guardia (de acuerdo a los reglamentos VIII/2 del Convenio STCW13 ${ }^{15}$ para mantener la supervisión general de la nave;

- Reglas de amarre y desamarre seguro de la nave;

- Garantizar el funcionamiento seguro de la embarcación cuando ésta realiza trabajo estacionario o casi estacionario en el mar;

- Prevención de la contaminación del medio ambiente marítimo;

- Mantener la seguridad y limpieza de todos los lugares para minimizar los riesgos de incendio;

- Proveer asistencia médica dentro de la embarcación;

- Garantizar la transportación segura de bienes;

- Mantenimiento e inspección de la integridad estructural de la nave;

- Actuar de conformidad con un Plan de Protección del Buque (Ship's Security $\left.P l a n^{16}\right)$ previamente aprobado;

La dotación mínima de seguridad de la embarcación también debe garantizar:

- Manejo y mantenimiento de todos los aparatos estancos y preservarlos en buen estado, así como la implementación de adecuados controles de daños.

14 De acuerdo con el párrafo 2 de la resolución, la implementación de estos principios a la administración marítima debe hacerse conforme a la regulación existente establecida por la Organización Marítima Internacional -IMO-, Organización Internacional del Trabajo -ILO-, Unión Internacional de Telecomunicaciones -ITU- y la Organización Mundial de la Salud -WHO- en lo relativo a: guardias por marineros, horas de trabajo y descanso, administración segura, certificación de marineros, entrenamiento, asistencia médica y alojamiento de la tripulación.

15 Regulación VIII/2 del Convenio Internacional sobre normas de formación, titulación y guardia para la gente del mar (STCW) de 1978 (enmendado en 1995 y 2010) concerniente a la organización de las guardias de los marineros y los principios a ser seguidos durante el ejercicio. 16 De acuerdo a la definición del punto 2 de la parte A del Código Internacional para la Protección de los Buques y de las instalaciones portuarias, un plan de protección del buque es un plan elaborado para asegurar la aplicación de medidas de seguridad en la nave para la protección de pasajeros, carga o catering y la propia unidad contra el riesgo de incidentes de seguridad 
- Mantenimiento respecto al equipo de combate de incendios, emergencias y equipo de salvamento, así como la capacidad de hacer reparaciones -si fuese necesario- en el mar.

- Mantenimiento de la maquinaria principal y auxiliar, así como la capacidad de mantenerlas en condiciones seguras como condición para que la nave pueda soportar potenciales peligros en su viaje.

\section{Conclusiones}

El problema de la fatiga, que se ha tratado en este trabajo, es sólo una de las muchas cuestiones relativas a la vida y el trabajo en el mar. La prevención de la fatiga en los barcos, así como la protección de la salud, seguridad, vida y propiedad, deben centrarse enormemente en entender las capacidades humanas y sus limitaciones. La información obtenida a través de un análisis profundo de la naturaleza humana debe utilizarse durante la creación de leyes y reglamentos atinentes a reglamentar el amplio espectro de actividad del hombre.

Por ello es importante que las autoridades legislativas, las instalaciones costeras y la tripulación de los barcos, intercambien información entre ellos y se mantengan en cooperación constante. La creación e implementación efectiva de regulaciones internacionales apropiadas en este sentido puede reportar muchos beneficios -reducir el número de errores cometidos, reducir los accidentes y víctimas en el mar, incrementar la productividad de las personas, ahorrar tiempo y reducir costos a los propietarios-.

Actualmente, la Organización Marítima Internacional tiene 170 Estados miembros, a saber, la vasta mayoría de países en el mundo que cuentan con una flota marítima ${ }^{17}$. Polonia, como miembro de la OMI desde 1960, así como muchos otros Estados miembros, ratifica e implementa en el derecho nacional previsiones contenidas en las convenciones internacionales (así como las 
directivas de la Unión Europea relativas a materias asociadas con el mar interior).

Por ello, concluimos que los problemas concernientes, por ejemplo, a la fatiga en los barcos, no surgen tanto de una ineficiente implementación de estas regulaciones en el derecho nacional o los Estados individualmente considerados, sino de errores cometidos en las naves, basados en una inadecuada regulación del derecho a las realidades que presenta la vida y el trabajo en el mar.

La necesidad de los dueños de barcos por cumplir los estándares internacionales sobre dotación mínima de seguridad (que no pueden ser evitados sin temer las consecuencias derivadas del incumplimiento de estándares jurídicos ${ }^{18}$ ), no contribuye tampoco a resolver el problema de la fatiga de la tripulación. Esto conduce a la conclusión de que los estándares internacionales sobre el tamaño de la tripulación están siendo drásticamente reducidos.

Por ello, un esfuerzo conjunto de todos los involucrados nos debe llevar a desarrollar estándares nacionales e internacionales apropiados y con ello alcanzar el máximo de eficiencia en la actividad humana que, al mismo tiempo, provea simultáneamente a cada persona una protección de sus derechos, seguridad y confort durante su vida y trabajo en el mar a la altura de los tiempos en que vivimos.

18 El cumplimiento en la nave de los convenios internacionales, incluyendo los estándares de dotación mínima de seguridad, puede ser revisado por el Control Portuario del Estado (Port State Control - PSC). En caso de múltiples incumplimientos, el PCS puede imponer penas al dueño e inclusive detener la nave en el puerto (lo cual acarrea muchas consecuencias negativas para las compañías como, por ejemplo, el incumplimiento de sus obligaciones). 


\section{Bibliografía y Webgrafia}

Committee on the Effect of Smaller Crews on Maritime Safety, Marine Board, Commission on Engineering and Technical Systems, National Research Council, op. cit., p. 85.

Convenio sobre el Trabajo Marítimo (MLC), 2006. Ratificado por Polonia (Diario Oficial - Dziennik Ustaw Rzeczypospolitej Polskiej-, núm. 222, disposición 1324) $\mathrm{y}$, por tanto, parte integrante del sistema jurídico polaco.

Convenio Internacional para la Seguridad de la Vida Humana en el Mar (SOLAS), 1974. Ratificado por Polonia (Diario Oficial - Dziennik Ustaw Rzeczypospolitej Polskiej-, 1984, núm. 61, disposición 318) y, por tanto, parte integrante del sistema jurídico polaco.

Corbett, Peter, A Modern Plague of Pirates, Reino Unido, Offshore and Marine Publications, 2009, p. 35; Committee on the Effect of Smaller Crews on Maritime Safety, Marine Board, Commission on Engineering and Technical Systems, National Research Council, Crew Size and Maritime Safety, Washington D.C. 1990, p. 1.

IMO, Member States, IGOs and NGOs, Disponible en internet: http://www.imo.org/About/Membership/Pages/Default.aspx. (Última consulta 1.03.2014)

IMO MSC/Circ. 1014, 12 de junio de 2001, Guidance on fatigue mitigation and management, Anexo "Guidelines on fatigue, Introduction, Foreword”, p. 1.

Łukaszuk, Leonard, "Międzynarodowa ochrona środowiska morskiego - głowne problemy, system regulacji oraz zasady i formy wspołpracy (zarys ogolny)", Studia Ecologiae et Bioethicae, Varsovia, núm. 1, 2003, p. 249. 
Marine Accident Investigation Branch, Annual report, 1999, Southampton, p. 5. Disponible en internet:

http://www.maib.gov.uk/cms_resources.cfm?file=/annual\%20report\%201999.pdf (Última consulta 15.02.2013)

Regulación VIII/2 del Convenio Internacional sobre normas de formación, titulación y guardia para la gente del mar (STCW) de 1978 (enmendado en 1995 y 2010) concerniente a la organización de las guardias de los marineros y los principios a ser seguidos durante el ejercicio.

Ross, Jonathan, Human factors for Naval Marine vehicle design and operation, Bodmin, Ashgate, 2009, p. 59).

Secretary-General performs depositary or other functions, 9 de enero de 2014, p. 18. Disponible en internet:

$<$ http://www.imo.org/About/Conventions/StatusOfConventions/Documents/Status \%20-\%202014\%20New\%20Version.pdf> (última consulta 1.03.2014). 